\title{
A lower bound for the error term in Weyl's law for certain Heisenberg manifolds
}

\author{
Werner Georg Nowak $\left.{ }^{*}\right)$ (Vienna)
}

\begin{abstract}
This article provides an Omega-result for the remainder term in Weyl's law for the spectral counting function of certain rational $(2 \ell+1)$-dimensional Heisenberg manifolds.
\end{abstract}

Introduction. For $M$ a closed $n$-dimensional Riemannian manifold with a metric $g$ and Laplace-Beltrami operator $\Delta$, let $N(t)$ denote the spectral counting function

$$
N(t):=\sum_{\substack{\lambda \text { eigenvalue of } \\ \lambda \leq t}} d(\lambda)
$$

where $d(\lambda)$ is the dimension of the eigenspace corresponding to $\lambda$, and $t$ is a large real variable. Then a deep and very general theorem due to L. Hörmander [6] tells us that

$$
N(t)=\frac{\operatorname{vol}(M)}{(4 \pi)^{n / 2} \Gamma\left(\frac{1}{2} n+1\right)} t^{n / 2}+O\left(t^{(n-1) / 2}\right)
$$

("Weyl's law"), and that the error term in general cannot be improved. Nevertheless, it is of interest to study the order of magnitude and the asymptotic behavior of the remainder $R(t)=N(t)-\frac{\operatorname{vol}(M)}{(4 \pi)^{n / 2} \Gamma\left(\frac{1}{2} n+1\right)} t^{n / 2}$ for special manifolds $M$.

The most classic example, namely the case that $M=\mathbb{R}^{n} / \mathbb{Z}^{n}$, the $n$-dimensional torus, is (equivalent to) a central problem in the theory of lattice points in large domains, namely to provide asymptotic results for the number $A_{n}(x)$ of integer points in an origin-centered $n$-dimensional ball of radius $x$, for any dimension $n \geq 2$. There exists a vast literature on this particular subject: We only refer to the works of Huxley [7], [8], Hafner [4], and Soundararajan [17] for the planar case, for the papers by Chamizo \& Iwaniec [1], HeathBrown [5], and Tsang [18] for dimension $n=3$, and to the monographs of Walfisz [20], and Krätzel [14], [15], as well as to the recent, quite comprehensive, survey article [9].

In fact, for $M=\mathbb{R}^{n} / \mathbb{Z}^{n}$, we see that ${ }^{(1)}\left\{\mathbf{u} \mapsto e(\mathbf{m} \cdot \mathbf{u}): \mathbf{m} \in \mathbb{Z}^{n}\right\}$ is a basis for the eigenfunctions of the Laplace operator $\Delta=-\sum_{j=1}^{n} \partial_{j j}$, acting on functions from $\mathbb{R}^{n} / \mathbb{Z}^{n}$

Mathematics Subject Classification (2000): 11N37, 35P20, 58J50, 11P21.

(*) The author gratefully acknowledges support from the Austrian Science Fund (FWF) under project Nr. P20847-N18.

(1) Bold face letters will denote throughout elements of $\mathbb{R}^{n}$, resp., $\mathbb{Z}^{n}$, which may be viewed also as $(1 \times n)$-matrices ("row vectors") where applicable. Further, $|\cdot|$ stands for the Euclidean norm. 
into $\mathbb{C}$. The corresponding eigenvalues are $4 \pi^{2}|\mathbf{m}|^{2}, \mathbf{m} \in \mathbb{Z}^{n}$. For any integer $k \geq 0$, let as usual $r_{n}(k)$ denote the number of ways to write $k$ as the sum of $n$ squares. Then, for each $k$ with $r_{n}(k)>0,4 \pi^{2} k$ is an eigenvalue of $\Delta$ whose eigenspace consists of all functions

$$
\mathbf{u} \mapsto \sum_{|\mathbf{m}|^{2}=k} c(\mathbf{m}) e(\mathbf{m} \cdot \mathbf{u}),
$$

where $c(\mathbf{m})$ are any complex coefficients. Its dimension obviously equals $r_{n}(k)$, hence

$$
N(t)=\sum_{k \geq 0: 4 \pi^{2} k \leq t} r_{n}(k)=A_{n}\left(\frac{\sqrt{t}}{2 \pi}\right) .
$$

2. Heisenberg manifolds. In recent times, presumably motivated by quite different areas like quantum physics and the abstract theory of PDE's, a lot of work has been done on another special case, namely that of so-called Heisenberg manifolds. To recall basics and to fix notions, let $\ell \geq 1$ be a given integer, and

$$
\gamma(\mathbf{x}, \mathbf{y}, z)=\left(\begin{array}{ccc}
1 & \mathbf{x} & z \\
{ }^{t} \mathbf{o}_{\ell} & I_{\ell} & { }^{t} \mathbf{y} \\
0 & \mathbf{o}_{\ell} & 1
\end{array}\right)
$$

where $\mathbf{x}, \mathbf{y} \in \mathbb{R}^{\ell}, z \in \mathbb{R}, \mathbf{o}_{\ell}=(0, \ldots, 0) \in \mathbb{R}^{\ell}, I_{\ell}$ is the $(\ell \times \ell)$-unit matrix, and ${ }^{t}$. denotes transposition. Then the $(2 \ell+1)$-dimensional Heisenberg group $H_{\ell}$ is defined by

$$
H_{\ell}=\left\{\gamma(\mathbf{x}, \mathbf{y}, z): \mathbf{x}, \mathbf{y} \in \mathbb{R}^{\ell}, z \in \mathbb{R}\right\}
$$

with the usual matrix product. Further, for any $\ell$-tuple $\mathbf{r}=\left(r_{1}, \ldots, r_{\ell}\right) \in \mathbb{Z}_{+}^{\ell}$ with the property that $r_{j} \mid r_{j+1}$ for all $j=1, \ldots, \ell-1$, we put $\mathbf{r} * \mathbb{Z}^{\ell}:=r_{1} \mathbb{Z} \times \ldots \times r_{\ell} \mathbb{Z}$ and define

$$
\Gamma_{\mathbf{r}}=\left\{\gamma(\mathbf{x}, \mathbf{y}, z): \mathbf{x} \in \mathbf{r} * \mathbb{Z}^{\ell}, \mathbf{y} \in \mathbb{Z}^{\ell}, z \in \mathbb{Z}\right\}
$$

$\Gamma_{\mathbf{r}}$ is a uniform discrete subgroup of $H_{\ell}$, i.e., the Heisenberg manifold $H_{\ell} / \Gamma_{\mathbf{r}}$ is compact. Fortunately, according to a deep work by Gordon and Wilson [2], Theorem 2.4, this seemingly quite special choice of $\Gamma_{\mathbf{r}}$ is in fact fairly general. The subgroups $\Gamma_{\mathbf{r}}$ classify all uniform discrete subgroups of $H_{\ell}$ up to automorphisms: For every uniform discrete subgroup $\Gamma$ of $H_{\ell}$ there exists a unique $\ell$-tuple $\mathbf{r}$ and an automorphism of $H_{\ell}$ which maps $\Gamma$ to $\Gamma_{\mathbf{r}}$.

However, to get a "rational" or "arithmetic" Heisenberg manifold - borrowing an expression due to Petridis \& Toth [16] - we have to make a quite particular choice of the metric involved $^{(2)}$. Following the example of [16], Theorem 1.1, and also Zhai [21], we pick

$$
g_{\ell}=\left(\begin{array}{cc}
I_{2 \ell} & { }^{t} \mathbf{o}_{2 \ell} \\
\mathbf{o}_{2 \ell} & 2 \pi
\end{array}\right) .
$$

(2) Compare the discussion below concerning the bound (3.3) valid for "almost all" metrics $g$. 
The spectrum of the Laplace-Beltrami operator on $H_{\ell} / \Gamma_{\mathbf{r}}$ has been analyzed in Gordon and Wilson [2], p. 259, and also in Khosravi and Petridis [12], p. 3564. It consists of two different classes $\mathcal{S}_{I}$ and $\mathcal{S}_{I I}$, where $\mathcal{S}_{I}$ is the spectrum of the Laplacian on the $2 \ell$-dimensional torus, and

$$
\mathcal{S}_{I I}=\left\{2 \pi\left(n_{0}^{2}+n_{0}\left(2 n_{1}+\ell\right)\right): n_{0} \in \mathbb{Z}^{+}, n_{1} \in \mathbb{Z}_{0}^{+}\right\}
$$

with multiplicities ( $=$ dimensions of corresponding eigenspaces) $2 n_{0}^{\ell} r_{1} \cdot \ldots \cdot r_{\ell}\left(\begin{array}{c}n_{1}+\ell-1 \\ \ell-1\end{array}\right)$.

3. Statement of problem and results. In this article, we shall be concerned with the size of the error term in (1.1) for the special case that $M=\left(H_{\ell} / \Gamma_{\mathbf{r}}, g_{\ell}\right)$ as described above, i.e., with the asymptotic behavior of

$$
R(t)=N(t)-\frac{\operatorname{vol}(M)}{(4 \pi)^{\ell+1 / 2} \Gamma\left(\ell+\frac{3}{2}\right)} t^{\ell+1 / 2}=N(t)-\frac{r_{1} \cdot \ldots \cdot r_{\ell}}{2^{2 \ell+1 / 2} \pi^{\ell} \Gamma\left(\ell+\frac{3}{2}\right)} t^{\ell+1 / 2} .
$$

For $\ell=1$, Petridis and Toth [16] proved that $R(t) \ll t^{5 / 6} \log t$. They were the first to realize that the question is related to a certain planar lattice point problem which can be dealt with usual tools for the estimation of fractional part sums. This result was sharpened and generalized to arbitrary $\ell \geq 1$ by Khosravi and Petridis [12] who obtained $R(t) \ll t^{\ell-7 / 41}$. Zhai [21] noticed that Huxley's deep method [7], [8] can be used to derive, for any $\ell \geq 1$,

$$
R(t) \ll t^{\ell-77 / 416}(\log t)^{26957 / 8320} .
$$

In fact, the difficulty in these estimations comes from the "rational" nature of the metric $g_{\ell}$. As Khosravi and Petridis [12] showed, for "almost all" metrics $g$ the much sharper bound

$$
R_{g}(t) \ll g t^{\ell-1 / 4} \log t
$$

holds true. Returning to the rational case (2.3), a result of Khosravi [11] and Khosravi \& Toth [13] tells us that

$$
\int_{0}^{T}(R(t))^{2} \mathrm{~d} t=C_{\ell} T^{2 \ell+1 / 2}+O\left(T^{2 \ell+1 / 4+\varepsilon}\right)
$$

where $C_{\ell}>0$ is an explicit constant. A recent paper of Zhai [21] provides estimates and asymptotics for higher power moments of $R(t)$. In fact, (3.3) and (3.4) may suggest the conjecture that

$$
R(t) \ll t^{\ell-1 / 4+\varepsilon}
$$

for every $\varepsilon>0$. The situation has a good deal in common with the Dirichlet divisor and the Gaussian circle problems. 
The objective of the present article is to provide a lower bound which shows that the $\varepsilon$ in (3.5) cannot be removed. We shall prove that

$$
R(t)=\Omega\left(t^{\ell-1 / 4}(\log t)^{1 / 4}\right)
$$

Together with (3.4), we may say that" $R(t) \ll t^{\ell-1 / 4}$ in mean-square, with an unbounded sequence of exceptionally large values $t$ ".

Unfortunately, we have to impose the restriction that $\ell$ is an even integer. We will comment on this condition at the end of the paper.

Theorem. For a fixed even positive integer $\ell$, let $\left(H_{\ell} / \Gamma_{\mathbf{r}}, g_{\ell}\right)$ be a rational $(2 \ell+1)$ dimensional Heisenberg manifold with metric $g_{\ell}$, as described above. Then the error term $R(t)$ for the associated spectral counting function, defined in (3.1), satisfies

$$
\limsup _{t \rightarrow \infty} \frac{R(t)}{t^{\ell-1 / 4}(\log t)^{1 / 4}}>0
$$

\section{Some Lemmas.}

Lemma 1. (Vaaler's approximation of fractional parts by trigonometric polynomials.) For arbitrary $w \in \mathbb{R}$ and $H \in \mathbb{Z}^{+}$, let $\psi(w):=w-[w]-\frac{1}{2}$,

$$
\Sigma_{H}(w):=\sum_{h=1}^{H} \alpha_{h, H} \sin (2 \pi h w), \quad \Sigma_{H}^{*}(w):=\sum_{h=1}^{H} \beta_{h, H} \cos (2 \pi h w)+\frac{1}{2 H+2}
$$

where, for $h=1, \ldots, H$,

$$
\alpha_{h, H}:=\frac{1}{\pi h} \rho\left(\frac{h}{H+1}\right), \quad \beta_{h, H}:=\frac{1}{H+1}\left(1-\frac{h}{H+1}\right),
$$

and

$$
\rho(\xi)=\pi \xi(1-\xi) \cot (\pi \xi)+\xi \quad(0<\xi<1) .
$$

Then the following inequality holds true:

$$
\left|\psi(w)+\Sigma_{H}(w)\right| \leq \Sigma_{H}^{*}(w)
$$

Proof. This is one of the main results in Vaaler [19]. A very well readable exposition can also be found in the monograph by Graham and Kolesnik [3]. 
Lemma 2. Let $F \in C^{4}[A, B], G \in C^{2}[A, B]$, and suppose that, for positive parameters $X, Y, Z$, we have $1 \ll B-A \ll X$ and

$$
F^{(j)} \ll X^{2-j} Y^{-1} \quad \text { for } j=2,3,4,\left|F^{\prime \prime}\right| \geq c_{0} Y^{-1}, \quad G^{(j)} \ll X^{-j} Z \quad \text { for } j=0,1,2,
$$

throughout the interval $[A, B]$, with some constant $c_{0}>0$. Let $\mathcal{J}^{\prime}$ denote the image of ] $A, B]$ under $F^{\prime}$, and $F^{*}$ the inverse function of $F^{\prime}$. Then, with $e(w)=e^{2 \pi i w}$ as usual,

$$
\begin{aligned}
\sum_{A<m \leq B} G(m) e(F(m))= & e\left(\frac{\operatorname{sgn}\left(F^{\prime \prime}\right)}{8}\right) \sum_{k \in \mathcal{J}^{\prime}} \frac{G\left(F^{*}(k)\right)}{\sqrt{\left|F^{\prime \prime}\left(F^{*}(k)\right)\right|}} e\left(F\left(F^{*}(k)\right)-k F^{*}(k)\right)+ \\
& +O\left(Z\left(\sqrt{Y}+\log \left(2+\operatorname{length}\left(\mathcal{J}^{\prime}\right)\right)\right)\right) .
\end{aligned}
$$

Proof. Transformation formulas of this kind are quite common, though often with worse error terms. This very sharp version can be found as f. (8.47) in the recent monograph [10] of H. Iwaniec and E. Kowalski.

Lemma 3. For a real parameter $T \geq 1$, let $\mathcal{F}_{T}$ denote the Fejér kernel

$$
\mathcal{F}_{T}(v)=T\left(\frac{\sin (\pi T v)}{\pi T v}\right)^{2}
$$

Then for arbitrary real numbers $Q>0$ and $\delta$, it follows that

$$
\int_{-1}^{1} \mathcal{F}_{T}(v) e(Q v+\delta) \mathrm{d} v=\max \left(1-\frac{Q}{T}, 0\right) e(\delta)+O\left(\frac{1}{Q}\right),
$$

where the $O$-constant is independent of $T$ and $\delta$.

Proof. This useful result is due to Hafner [4]. It follows from the classic Fourier transform formula

$$
\int_{\mathbb{R}} \mathcal{F}_{T}(v) e(Q v) \mathrm{d} v=\int_{\mathbb{R}}\left(\frac{\sin (\pi v)}{\pi v}\right)^{2} e\left(\frac{Q}{T} v\right) \mathrm{d} v=\max \left(1-\frac{Q}{T}, 0\right) .
$$

Since $\mathcal{F}_{T}( \pm 1) \ll T^{-1}$ and $\mathcal{F}_{T}^{\prime}(v) \ll v^{-2}$ for $|v| \geq 1$, uniformly in $T \geq 1$, integration by parts readily shows that the intervals $]-\infty,-1]$ and $\left[1, \infty\left[\right.\right.$ contribute only $O\left(Q^{-1}\right)$.

5. Proof of the Theorem. We start from Lemma 3.1 in Zhai [21] which approximates the error term involved by a fractional part sum. Let $U$ be a large real parameter, $u \in[U-1, U+1]$, and put

$$
E(u):=\frac{2^{\ell-2}(\ell-1) !}{r_{1} \cdot \ldots \cdot r_{\ell}} R\left(2 \pi u^{2}\right)
$$


Then according to Zhai ${ }^{(3)}$ [21], Lemma 3.1, for $\ell$ even,

$$
\begin{aligned}
E(u) & =E^{*}(u)+O\left(u^{2 \ell-1}\right), \\
E^{*}(u) & :=-\sum_{1 \leq m \leq u} m\left(u^{2}-m^{2}\right)^{\ell-1} \psi\left(\frac{u^{2}}{2 m}-\frac{m}{2}\right) .
\end{aligned}
$$

We apply Lemma 1 in the form $-\psi \geq \Sigma_{H}-\Sigma_{H}^{*}$, choosing $H=[U]$. Thus we get

$$
\begin{aligned}
E^{*}(u) \geq & -U+\sum_{1 \leq h \leq U} \sum_{1 \leq m \leq u} m\left(u^{2}-m^{2}\right)^{\ell-1} \times \\
& \times\left(\alpha_{h,[U]} \sin \left(2 \pi h\left(\frac{u^{2}}{2 m}-\frac{m}{2}\right)\right)-\beta_{h,[U]} \cos \left(2 \pi h\left(\frac{u^{2}}{2 m}-\frac{m}{2}\right)\right)\right) .
\end{aligned}
$$

We split up the range $1 \leq m \leq u$ into dyadic subintervals $\left.\left.\mathcal{M}_{j}=\right] M_{j+1}, M_{j}\right], M_{j}=u 2^{-j}$ for $j=0, \ldots, J$, where $J$ is minimal such that $(U-1) 2^{-J-1}<1$. We thus have to deal with exponential sums

$$
\mathcal{E}_{j}(h, u):=\sum_{m \in \mathcal{M}_{j}} m\left(u^{2}-m^{2}\right)^{\ell-1} e\left(-h\left(\frac{u^{2}}{2 m}-\frac{m}{2}\right)\right)
$$

We transform them by means of Lemma 2, with

$$
G(\xi)=\xi\left(u^{2}-\xi^{2}\right)^{\ell-1}, \quad F(\xi)=-h\left(\frac{u^{2}}{2 \xi}-\frac{\xi}{2}\right)
$$

By straightforward computations, on each interval $\mathcal{M}_{j}$ the conditions of Lemma 2 are fulfilled with the parameters $X=M_{j}, Y=\frac{M_{j}^{3}}{h u^{2}}, Z=M_{j} u^{2 \ell-2}$. We obtain

$$
\begin{aligned}
\mathcal{E}_{j}(h, u)= & h^{3 / 4} u^{2 \ell-1 / 2} \sum_{k \in F^{\prime}\left(\mathcal{M}_{j}\right)} \frac{(2 k-2 h)^{\ell-1}}{(2 k-h)^{\ell+1 / 4}} e\left(-u \sqrt{h} \sqrt{2 k-h}-\frac{1}{8}\right) \\
& +O\left(u^{2 \ell-3} \frac{M_{j}^{5 / 2}}{h^{1 / 2}}+u^{2 \ell-1} \log u\right) .
\end{aligned}
$$

We first bound the overall contribution of the error terms, summing over $j$ and $h$. Let $\gamma_{h,[U]}$ denote $\alpha_{h,[U]}$ or $\beta_{h,[U]}$, thus $\gamma_{h,[U]} \ll h^{-1}$ in any case, then

$$
\sum_{j=0}^{J} \sum_{h=1}^{[U]} \gamma_{h,[U]}\left(u^{2 \ell-3} \frac{M_{j}^{5 / 2}}{h^{1 / 2}}+u^{2 \ell-1} \log u\right) \ll u^{2 \ell-1 / 2}+u^{2 \ell-1}(\log u)^{3} \ll u^{2 \ell-1 / 2}
$$

(3) In fact, Zhai in his notation tacitly assumes that $r_{1}=\ldots=r_{\ell}=1$, which means no actual loss of generality. We have supplemented the factor $r_{1} \cdot \ldots \cdot r_{\ell}$ in (5.1). 
Summing up the main terms in (5.4), we notice that the total range of $k$ becomes $h=F^{\prime}\left(M_{0}\right)<k \leq F^{\prime}\left(M_{J+1}\right)=\frac{1}{2} h+2^{2 J+1} h=: K_{h, U}$, and we obtain

$$
\begin{aligned}
& \sum_{1 \leq h \leq U} \sum_{1 \leq m \leq u} m\left(u^{2}-m^{2}\right)^{\ell-1} \gamma_{h,[U]} e\left(-h\left(\frac{u^{2}}{2 m}-\frac{m}{2}\right)\right)= \\
& =u^{2 \ell-1 / 2} \sum_{h=1}^{[U]} \gamma_{h,[U]} h^{3 / 4} \sum_{h<k \leq K_{h, U}} \frac{(2 k-2 h)^{\ell-1}}{(2 k-h)^{\ell+1 / 4}} e\left(-u \sqrt{h} \sqrt{2 k-h}-\frac{1}{8}\right)+ \\
& +O\left(u^{2 \ell-1 / 2}\right) .
\end{aligned}
$$

Using the real and imaginary part of this result in (5.3), we arrive at

$$
E^{*}(u) \geq u^{2 \ell-1 / 2} S(u, U)-c_{1} u^{2 \ell-1 / 2}
$$

where

$$
\begin{aligned}
S(u, U):= & \sum_{(h, k) \in \mathcal{D}(U)} h^{3 / 4} \frac{(2 k-2 h)^{\ell-1}}{(2 k-h)^{\ell+1 / 4}} \times \\
& \times\left(\alpha_{h,[U]} \sin \left(2 \pi u \sqrt{h} \sqrt{2 k-h}+\frac{\pi}{4}\right)-\beta_{h,[U]} \cos \left(2 \pi u \sqrt{h} \sqrt{2 k-h}+\frac{\pi}{4}\right)\right), \\
& \mathcal{D}(U):=\left\{(h, k) \in \mathbb{Z}^{2}: 1 \leq h \leq U, h<k \leq K_{h, U}\right\},
\end{aligned}
$$

and $c_{1}$ is an appropriate positive constant. Our next step is to get rid of "most" of the terms of the last double sum. To this end we use Lemma 3, multiplying $S(u, U)$ by a Fejér kernel $\mathcal{F}_{T}(u-U)$, where $T$ is a new large parameter, and integrating over $U-1 \leq u \leq U+1$. We obtain

$$
\begin{aligned}
& I(T, U):=\int_{U-1}^{U+1} S(u, U) \mathcal{F}_{T}(u-U) \mathrm{d} u=\int_{-1}^{1} S(U+v, U) \mathcal{F}_{T}(v) \mathrm{d} v= \\
& =\sum_{(h, k) \in \mathcal{D}(U), h(2 k-h) \leq T^{2}} h^{3 / 4} \frac{(2 k-2 h)^{\ell-1}}{(2 k-h)^{\ell+1 / 4}}\left(1-\frac{\sqrt{h(2 k-h)}}{T}\right) \times \\
& \times\left(\alpha_{h,[U]} \sin \left(2 \pi U \sqrt{h(2 k-h)}+\frac{\pi}{4}\right)-\beta_{h,[U]} \cos \left(2 \pi U \sqrt{h(2 k-h)}+\frac{\pi}{4}\right)\right) \\
& +O\left(\sum_{(h, k) \in \mathcal{D}(U)} h^{-3 / 4} \frac{(2 k-2 h)^{\ell-1}}{(2 k-h)^{\ell+3 / 4}}\right) .
\end{aligned}
$$

The $O$-term here is harmless: In fact,

$$
\sum_{h=1}^{\infty} h^{-3 / 4} \sum_{k>h} \frac{(2 k-2 h)^{\ell-1}}{(2 k-h)^{\ell+3 / 4}} \ll \sum_{h=1}^{\infty} h^{-3 / 4} \sum_{m>h / 2} m^{-7 / 4} \ll \sum_{h=1}^{\infty} h^{-3 / 2} \ll 1
$$


We proceed to derive a lower bound for the main term in (5.8). To this end we relate the two parameters $U$ and $T$ to each other: For arbitrary $T$ sufficiently large, we choose $U$ according to the Dirichlet approximation theorem, such that

$$
T^{2} \leq U \leq T^{2} 16^{T^{2}}
$$

and

$$
\|U \sqrt{h(2 k-h)}\| \leq \frac{1}{16}
$$

for all $(h, k) \in \mathcal{D}(U), h(2 k-h) \leq T^{2}$, where $\|\cdot\|$ denotes the distance from the nearest integer. Now $h(2 k-h) \leq T^{2}, k>h$, together with (i) implies that

$$
h \leq T \leq \sqrt{U}
$$

By the definitions in Lemma 1, for all of the $h$ occurring in (5.8), $\alpha_{h,[U]} \asymp h^{-1}$, and $\beta_{h,[U]} \asymp U^{-1}$, hence

$$
\begin{aligned}
\alpha_{h,[U]} & \sin \left(2 \pi U \sqrt{h(2 k-h)}+\frac{\pi}{4}\right)-\beta_{h,[U]} \cos \left(2 \pi U \sqrt{h(2 k-h)}+\frac{\pi}{4}\right) \geq \\
& \geq c_{1} \sin \left(\frac{\pi}{8}\right) \frac{1}{h}-\frac{c_{2}}{U} \geq \frac{c_{3}}{h},
\end{aligned}
$$

with suitable positive constants $c_{1}, c_{2}, c_{3}$. Putting for short

$$
\theta_{U, T, \ell}(n):=\sum_{(h, k) \in \mathcal{D}(U), h(2 k-h)=n} \frac{h^{1 / 2}}{(2 k-h)^{1 / 2}}\left(1-\frac{h}{2 k-h}\right)^{\ell-1}
$$

we thus readily infer from (5.8) and (5.9) that, for some $c_{4}, c_{5}>0$,

$$
\begin{aligned}
I(T, U) & \geq c_{3} \sum_{1 \leq n \leq T^{2}} \frac{\theta_{U, T, \ell}(n)}{n^{3 / 4}}\left(1-\frac{\sqrt{n}}{T}\right)-c_{4} \\
& \geq c_{5} \sum_{1 \leq n \leq T^{2} / 2} \frac{\theta_{U, T, \ell}(n)}{n^{3 / 4}}-c_{4} \cdot
\end{aligned}
$$

We notice further that $(h, k) \in \mathcal{D}(U)$ explicitly means that

$$
1 \leq h \leq U, \quad h<k \leq \frac{1}{2} h+2^{2 J+1} h \asymp U^{2} h,
$$

while $h(2 k-h) \leq T^{2}, k>h$ implies (5.10) and

$$
k<2 k-h \leq \frac{T^{2}}{h} \leq T^{2} \leq U .
$$


Hence, for $1 \leq n \leq T^{2}$,

$$
\begin{gathered}
\theta_{U, T, \ell}(n)=\theta_{\ell}(n):=\sum_{\substack{h(2 k-h)=n \\
k>h}} \frac{h^{1 / 2}}{(2 k-h)^{1 / 2}}\left(1-\frac{h}{2 k-h}\right)^{\ell-1} \\
\gg \sum_{\substack{h(2 k-h)=n \\
k>2 h}} \frac{h^{1 / 2}}{(2 k-h)^{1 / 2}} \gg \sum_{\substack{h m=n, m>3 h \\
h \equiv m \bmod 2}} \frac{\sqrt{h}}{\sqrt{m}} \gg \sum_{\substack{h m=n, 3 h<m \leq 4 h \\
h \equiv m \bmod 2}} 1 .
\end{gathered}
$$

Therefore, by (5.11),

$$
\begin{aligned}
& I(T, U)+c_{4} \sum_{\substack{T^{2} / 4 \leq n \leq T^{2} / 2 \\
n \text { odd }}} n^{-3 / 4} \sum_{\substack{h m=n \\
3 h<m \leq 4 h}} 1 \\
& \gg T^{-3 / 2} \sum_{\substack{T^{2} / 4 \leq h m \leq T^{2 / 2} \\
3 h<m \leq 4 h, h, m \text { odd }}} 1 \gg T^{1 / 2} .
\end{aligned}
$$

Thus,

$$
I(T, U) \gg T^{1 / 2} \gg(\log U)^{1 / 4},
$$

since (i) readily implies that $T \gg(\log U)^{1 / 2}$. On the other hand, it follows from the definition of $I(T, U)$ that

$$
I(T, U) \leq\left(\sup _{U-1 \leq u \leq U+1} S(u, U)\right) \int_{-1}^{1} \mathcal{F}_{T}(v) \mathrm{d} v .
$$

Since

$$
\int_{-1}^{1} \mathcal{F}_{T}(v) \mathrm{d} v=\int_{-T}^{T}\left(\frac{\sin (\pi v)}{\pi v}\right)^{2} \mathrm{~d} v \leq 1,
$$

this implies that there exists a value $u^{*} \in[U-1, U+1]$ for which

$$
S\left(u^{*}, U\right) \geq c_{6}\left(\log u^{*}\right)^{1 / 4},
$$

$c_{6}$ a suitable positive constant. It remains to recall that if $T$ runs through an unbounded sequence of positive reals, by construction so do $U$ and $u^{*}$. Therefore, (5.1), (5.2), (5.7) and (5.12) together complete the proof of our theorem.

6. Concluding remarks. 1 . It is appropriate to comment on the somewhat disturbing restriction that $\ell$ must be even. In general, in (5.2) the argument of the function $\psi$ contains an additional term $-\frac{\ell}{2}$, according to Zhai's [21] Lemma 3.1. This gives an additional factor $e\left(\frac{1}{2} h \ell\right)$ at the right-hand side of (5.6), and as a consequence, additional terms $\pi h \ell$ in the arguments of the sine and cosine in (5.8). For $\ell$ odd, the definitions of $\theta_{U, T, \ell}(n)$ and $\theta_{\ell}(n)$ ultimately contain alternating factors $(-1)^{h}$ which fatally affect our argument, at least in its present form. 
2. It is a natural question whether the sophisticated new methods due to Hafner [4] and Soundararajan [17] can be applied, in order to improve the result by a loglog-factor, as it is the case for the divisor and circle problems. However, both arguments are based on the fact that for $d(n)$ and $r(n)$, the average order is accomplished by a "thin" set of integers on which $d(n)$, resp., $r(n)$ attain exceptionally large values. The improvement is effected by restricting the application of the Dirichlet approximation theorem to such a thin set. In our present case, a similar observation concerning the arithmetic function $\theta_{\ell}(n)$ is at least not at all straightforward, inter alia because $\theta_{\ell}(n)$ fails to be multiplicative.

\section{References}

[1] F. Chamizo and H. Iwaniec, On the sphere problem. Rev. Mat. Iberoamericana 11, 417-429 (1995).

[2] C.S. Gordon and E.N. Wilson, The spectrum of the Laplacian on Riemannian Heisenberg manifolds, Michigan Math. J. 33, 253-271 (1986).

[3] S.W. Graham and G. Kolesnik, Van der Corput's method on exponential sums, Cambridge 1991.

[4] J.L. Hafner, New omega results for two classical lattice point problems, Invent. Math. 63, 181-186 (1981).

[5] D.R. Heath-Brown, Lattice points in the sphere, In: Number theory in progress, Proc. Number Theory Conf. Zakopane 1997, eds. K. Györy et al., vol. 2 (1999), 883-892.

[6] L. Hörmander, The spectral function of an elliptic operator, Acta Math. 121, 193-218 (1968).

[7] M.N. Huxley, Area, lattice points, and exponential sums. LMS Monographs, New Ser. 13, Oxford 1996.

[8] M.N. Huxley, Exponential sums and lattice points III. Proc. London Math. Soc. (3) 87, 591-609 (2003).

[9] A. Ivić, E. Krätzel, M. Kühleitner, and W.G. Nowak, Lattice points in large regions and related arithmetic functions: Recent developments in a very classic topic. Proceedings Conf. on Elementary and Analytic Number Theory ELAZ'04, held in Mainz, May 24-28, W. Schwarz and J. Steuding eds., Franz Steiner Verlag 2006, pp. 89-128.

[10] H. Iwaniec, E. Kowalski, Analytic Number Theory, AMS Coll.Publ. 53. Providence, R.I., 2004.

[11] M. Khosravi, Spectral statistics for Heisenberg manifolds, Ph.D. thesis, McGill U. 2005.

[12] M. Khosravi and Y.N. Petridis, The remainder in Weyl's law for $n$-dimensional Heisenberg manifolds, Proc. AMS 133/12, 3561-3571 (2005).

[13] M. Khosravi and J.A. Toth, Cramer's formula for Heisenberg manifolds, Ann. de l'institut Fourier 55, 2489-2520 (2005).

[14] E. Krätzel, Lattice points. Berlin 1988.

[15] E. Krätzel, Analytische Funktionen in der Zahlentheorie. Stuttgart-Leipzig-Wiesbaden 2000.

[16] Y.N. Petridis and J.A. Toth, The remainder in Weyl's law for Heisenberg manifolds, J. Diff. Geom. 60, 455-483 (2002).

[17] K. Soundararajan, Omega results for the divisor and circle problems. Int. Math. Res. Not. 36, 1987-1998 (2003).

[18] K.-M. Tsang, Counting lattice points in the sphere. Bull. London Math. Soc. 32, 679-688 (2000).

[19] J.D. Vaaler, Some extremal problems in Fourier analysis, Bull. Amer. Math. Soc. 12, 183-216 (1985).

[20] A. Walfisz, Gitterpunkte in mehrdimensionalen Kugeln, Warszaw 1957.

[21] W. Zhai, On the error term in Weyl's law for the Heisenberg manifolds, Acta Arithm. 134, 219-257 (2008).

Institute of Mathematics

Department of Integrative Biology

Universität für Bodenkultur Wien

Gregor Mendel-Straße 33

1180 Wien, Österreich

E-mail: nowak@boku.ac.at 